\title{
Role of electron-phonon interactions versus electron-electron interactions in the broadening mechanism of the electron and hole linewidths in bulk Be
}

\author{
I. Yu. Sklyadneva, ${ }^{1}$ E. V. Chulkov, ${ }^{1,2, *}$ W.-D. Schöne, ${ }^{3,4}$ V. M. Silkin, ${ }^{1}$ R. Keyling, ${ }^{1}$ and P. M. Echenique ${ }^{1,2}$ \\ ${ }^{1}$ Donostia Internation Physics Center (DIPC), 20018 San Sebastián/Donostia, Basque Country, Spain \\ ${ }^{2}$ Departamento de Física de Materiales and Centro Mixto CSIC-UPV/EHU, Facultad de Ciencias Químicas, UPV/EHU, Apdo. 1072, \\ 20080 San Sebastián/Donostia, Basque Country, Spain \\ ${ }^{3}$ Fritz-Haber-Institut der Max-Planck-Gesellschaft, Faradayweg 4-6, 14195 Berlin, Germany \\ ${ }^{4}$ Freie Universität Berlin, Fachbereich Physik, Arnimallee 14, 14195 Berlin, Germany
}

(Received 18 November 2004; published 20 May 2005)

\begin{abstract}
We report on $a b$ initio study of electron-phonon $(e-\mathrm{ph})$ and electron-electron $(e-e)$ interactions in bulk Be. The calculations show that the $e$-ph coupling parameter $\lambda$ varies from 0.01 to 1.02 as a function of electron energy and momentum, $\lambda=0.21$ at the Fermi level as averaged over momenta. The $e$-ph contribution $\Gamma_{e \text {-ph }}$ to the electrons and holes lifetime broadening also manifests clear dependence on the momentum and energy of an electron state. We demonstrate that the $e$-ph coupling matrix elements strongly affect the Eliashberg function especially for low phonon frequencies. By using the Debye model relation between $\Gamma_{e \text {-ph }}$ and $\lambda$ the characteristic Debye frequency $\omega_{D}$ is obtained in good agreement with the experimental one. The $e-e$ contribution to the lifetime broadening is evaluated from the imaginary part of the electron self-energy computed within a GW approximation.
\end{abstract}

DOI: 10.1103/PhysRevB.71.174302

PACS number(s): 71.45.Gm, 79.20.Uv, 71.15.Mb, 71.15.Dx

\section{INTRODUCTION}

Electron-phonon $(e-\mathrm{ph})$ interaction is important for the description of many phenomena in condensed matter physics. In particular, it is a cornerstone of conventional superconductivity and plays a key role in the description of electrical and thermal resistivity, renormalization of electron bands close to the Fermi level $\left(E_{F}\right)$, structural phase transitions and charge density waves. ${ }^{1}$ It also contributes to the final lifetimes of excited electrons and holes, ${ }^{2,3}$ being especially important for energies close to $E_{F}$. The strength of the $e$-ph interaction is described by an $e$-ph coupling parameter $\lambda$, which depends on both the electron (hole) energy $\epsilon_{\mathrm{k}}$ and momentum $\mathbf{k}$. In the past, $\lambda$ was mostly obtained from tunneling or specific heat measurements ${ }^{1}$ at $E_{F}$. Recently photoemission spectroscopy (PES) was successfully applied to deduce $\lambda$ from the temperature dependence of the measured hole linewidth in surface states on metal surfaces. ${ }^{4-12}$ In some PES experiments $\lambda$ was obtained from the measured slope of the real part of the self-energy at $E_{F} \cdot{ }^{13,14}$ Later PES was also used to measure $\lambda$ for quantum well states in adlayers on metal substrates. ${ }^{15-20}$ Despite its unprecedented accuracy of measuring $\lambda$ for surface and quantum well states PES is not well suited to measure true hole lifetimes for bulk materials because of the known final states effect. ${ }^{21}$ In principle, the energy resolved $\lambda$ for bulk states can be obtained from photoemission measurements of quantum well states for very thick adlayer films. However, it can be done only for a symmetry direction in the bulk Brillouin zone which is perpendicular to the adfilm.

In this situation valuable information on $e$-ph interactions can be obtained from theoretical calculations, especially from $a b$ initio computations, since such calculations can be done for any wave vector in the Brillouin zone. The electronphonon interaction is fully described by the Eliashberg spec- tral function $\alpha^{2} F(w)$ and its first reciprocal moment $\lambda .^{22,23}$ The evaluation of these basic quantities requires the knowledge of the electron energy spectrum, the complete phonon spectrum and the changes in the effective crystal potential upon lattice vibrations.

In this work we calculate the Eliashberg function $\alpha^{2} F(w)$, the $e$-ph coupling parameter $\lambda$, and the phonon induced contribution to the excited electron and hole inverse lifetimes $\tau^{-1}$ for bulk Be. This metal has been chosen for two reasons. First, bulk Be electron bands show very anisotropic behavior that can seriously affect $e$-ph interactions as well as quasiparticle lifetimes. ${ }^{24}$ Second, electronic properties of bulk Be are very distinct from those in Be surfaces. For instance, the electron density of states (DOS) in bulk Be has a very deep minimum at $E_{F}$ making this material nearly semiconducting (see Ref. 25 and Fig. 1 below) while in the $\mathrm{Be}(0001)$ and $\operatorname{Be}(10 \overline{1} 0)$ surfaces the DOS at $E_{F}$ was found to be larger by a factor of 4-5 that makes these surfaces two-dimensional free-electron-like metals. ${ }^{26-28}$ The distinct character of the electronic structure can result in different $e$-ph interactions in the bulk and in the surfaces. Indeed, recent photoemission measurements of $\lambda$ gave $\lambda=0.7$ for the $\bar{\Gamma}$ surface state on $\mathrm{Be}(0001)$ (Ref. 14) at $E_{F}$ and $\lambda=0.66$ for the $\bar{A}$ surface state on $\mathrm{Be}(10 \overline{1} 0)$ (Refs. 9 and 11) that are significantly larger than the theoretical $\lambda=0.24$ evaluated long ago at $E_{F}$ for bulk Be (Ref. 1) (we are not aware of any measurement of $\lambda$ in bulk $\mathrm{Be})$. Recent $a b$ initio calculation ${ }^{29}$ of $\mathrm{Be}(0001)$ also gave a large $\lambda$ value confirming stronger $e$-ph interaction in surfaces. Despite a wealth of data on $e$-ph interactions in Be surfaces for bulk Be there are only estimates of $\lambda$ at $E_{F}$ based on semiempirical relations to the superconducting transition temperature $T_{c}(\lambda=0.24 \pm 0.01)$ (Refs. 1, 30, and 31) and pseudopotential calculations $(\lambda=0.26) .{ }^{32}$

Here we present the $a b$ initio calculation results in detail 


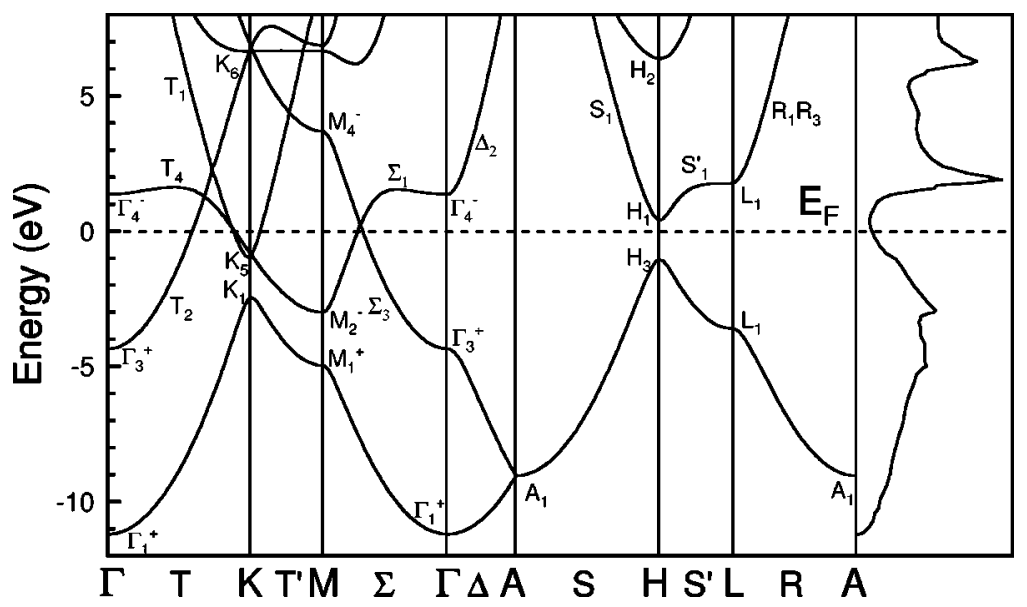

FIG. 1. LDA energy bands along the symmetry directions and density of electron states (right panel) for hcp Be.

for $\alpha^{2} F(w), \lambda$, and $\tau_{e-\mathrm{ph}}^{-1}=\Gamma_{e \text {-ph }}$ resolved in energy and momentum. We show that $\lambda$ varies from 0.01 at the $\Gamma_{1}^{+}$state to 1.02 at the unoccupied $H_{1}$ state, i.e., the maximum $\lambda$ value is larger than the minimum one by two orders of magnitude. The phonon induced contribution to $\tau^{-1}$ also experiences noticeable variations from a few meV to nearly one hundred meV. Strong impact of electron-phonon coupling matrix elements on $\alpha^{2} F(w)$ is found. In particular, the matrix elements completely suppress low-frequency peaks of the phonon density of states. To complete the study of electron and hole decay mechanisms we also present here the calculation results for an electron-electron $(e-e)$ contribution $\tau_{e-e}^{-1}=\Gamma_{e-e}$. The $\Gamma_{e-e}$ has been evaluated within a full $a b$ initio method from the imaginary part of the electron self-energy of the excited quasiparticles using the so-called $G W$ approximation of many-body theory. ${ }^{33}$ Previously the $\Gamma_{e-e}$ in bulk Be has been computed by using an "energy-shell" approximation for excited electrons only. ${ }^{24}$ Here we calculate $\Gamma_{e-e}$ from the imaginary part of the self-energy for both the electron and hole excitations at the quasiparticle energy. Finally we evaluate the full linewidth $\Gamma_{\text {tot }}=\Gamma_{e-\mathrm{ph}}+\Gamma_{e-e}$.

The paper is organized as follows. A short outline of the calculation methods is given in Sec. II. In Sec. III we present and discuss in detail the calculation results. Finally the conclusions are drawn in Sec. IV.

\section{THEORY}

\section{A. Lifetime due to electron-phonon scattering}

The phonon-induced linewidth $\Gamma_{e \text {-ph }}\left(\epsilon_{\mathbf{k}, i}\right)$ or lifetime $\tau_{e \text {-ph }}\left(\epsilon_{\mathbf{k}, i}\right)$ of an electron state with initial momentum $\mathbf{k}_{i}$ and energy $\epsilon_{\mathbf{k}, i}$ can be evaluated from the imaginary part of the electron-phonon self-energy which is related to the Eliashberg spectral function through the integral over all the scattering events that conserve energy and momentum:

$$
\begin{aligned}
\hbar / \tau_{e-\mathrm{ph}}\left(\epsilon_{\mathbf{k}, i}\right)= & \Gamma_{e-\mathrm{ph}}\left(\epsilon_{\mathbf{k}, i}\right)=2 \hbar \operatorname{Im} \Sigma_{i}^{0}\left(\epsilon_{\mathbf{k}, i} ; T\right) \\
= & 2 \pi \hbar \int_{0}^{w_{m}} \alpha^{2} F_{\mathbf{k}, i}(w)\left[1-f\left(\epsilon_{\mathbf{k}, i}-w\right)+f\left(\epsilon_{\mathbf{k}, i}+w\right)\right. \\
& +2 n(w)] d w .
\end{aligned}
$$

Here, $f(w)$ and $n(w)$ are the Fermi and Bose distributions, respectively, which introduce a temperature dependence of $\Gamma_{e-\mathrm{ph}}, w_{m}$ is the maximum phonon frequency, and

$$
\alpha^{2} F_{\mathbf{k}, i}(w)=\sum_{\mathbf{q}, \nu, f} \delta\left(w-w_{\mathbf{q}, \nu}\right)\left|g\left(\mathbf{k}_{i}, \mathbf{k}_{f}, \mathbf{q}, \nu\right)\right|^{2} \delta\left(\epsilon_{\mathbf{k}, i}-\epsilon_{\mathbf{k}, f}\right)
$$

is the Eliashberg spectral function for the particular electron state $\mathbf{k}_{i}=(\mathbf{k}, i)$, with $\mathbf{k}$ being a wave vector in the Brillouin zone (BZ) and $i$ a band index. The sum in Eq. (2) is over final electron states $\mathbf{k}_{f}$ and all possible phonon modes $\nu$ with momentum q. In Eq. (2) we apply the commonly used quasielastic scattering approximation $\delta\left(\epsilon_{\mathbf{k}, i}-\epsilon_{\mathbf{k}, f} \pm w_{\mathbf{q}, \nu}\right) \approx \delta\left(\epsilon_{\mathbf{k}, i}\right.$ $\left.-\epsilon_{\mathbf{k}, f}\right)$, which allows us to calculate $\Gamma_{e \text {-ph }}$ with the same Eliashberg spectral function for both emission and absorption processes. The electron-phonon matrix element $g\left(\mathbf{k}_{i}, \mathbf{k}_{f}, \mathbf{q}, \nu\right)$ reflects the probability of electron scattering from the initial state $\mathbf{k}_{i}$ to the final state $\mathbf{k}_{f}$ by the phonon $\mathbf{q}_{\nu}$.

The electron-state-dependent strength of the electronphonon coupling is measured by the dimensionless parameter $\lambda$, defined as the first inverse frequency moment of the spectral function ${ }^{1}$

$$
\lambda_{\mathbf{k}, i}=2 \int_{0}^{w_{m}} \frac{\alpha^{2} F_{\mathbf{k}, i}(w)}{w} d w .
$$

One can average the spectral function $\alpha^{2} F_{\mathbf{k}, i}(w)$ over the initial states on the Fermi surface defined by $\epsilon_{\mathbf{k}, i}=E_{F}$ :

$$
\begin{aligned}
\alpha^{2} F(w)= & \frac{1}{N\left(E_{F}\right)} \sum_{\mathbf{q}, \nu} \delta\left(w-w_{\mathbf{q}, \nu}\right) \sum_{\mathbf{k}, i, f}\left|g\left(\mathbf{k}_{i}, \mathbf{k}_{f}, \mathbf{q}, \nu\right)\right|^{2} \\
& \times \delta\left(\epsilon_{\mathbf{k}, i}-E_{F}\right) \delta\left(\epsilon_{\mathbf{k}, f}-E_{F}\right),
\end{aligned}
$$

where $N\left(E_{F}\right)$ is the electron density of states per atom and per spin at the Fermi level $E_{F}$. In this case we obtain the spectral function and the corresponding electron-phonon coupling parameter $\lambda$ as the Fermi surface averaged quantities.

All the self-consistent calculations of phonons and electron-phonon interaction presented here have been performed using the density-functional perturbation theory ${ }^{34}$ (DFPT) and the PWSCF code. ${ }^{35}$ We employed a nonlocal norm-conserving pseudopotential ${ }^{36}$ to describe the electron- 
ion interaction in Be and a plane-wave basis set to expand the crystal wave functions. The basis set was restricted by the kinetic energy cutoff of $22 \mathrm{Ry}$, which is enough to obtain the convergence within a few percent for the calculated phonon frequencies. Local density approximation (LDA) is used for the exchange and correlation energy functional in the Perdew-Zunger form. ${ }^{37}$

\section{B. Lifetime due to electron-electron scattering}

The calculation of the $e$-e contribution to the lifetime of excited electrons or holes from first principles is by now an established technique. ${ }^{38-42}$ Therefore only a short summary of the formalism used is given here. For more details one can see Ref. 38. The first step is a well-converged solution of the ground state of the system under consideration utilizing the Kohn-Sham (KS) equations ${ }^{43}$ of density-functional theory. For a crystalline system the KS equations are given by

$$
\left(-\frac{\hbar^{2}}{2 m} \nabla^{2}+v_{\text {eff }}(\mathbf{r})\right) \varphi_{\mathbf{k}, i}(\mathbf{r})=\epsilon_{\mathbf{k}, i} \varphi_{\mathbf{k}, i}(\mathbf{r}),
$$

where $v_{\text {eff }}(\mathbf{r})$ is the mean-field potential in which the KS electrons move. It already contains exchange-correlation interaction (for instance, at the LDA level) which does not include any dynamical effects in order to describe the lifetime of excited states. These effects are included via a renormalization, which leads to the Dyson equation ${ }^{44,45}$

$$
\begin{aligned}
& {\left[-\frac{\hbar^{2}}{2 m} \nabla^{2}+v_{\mathrm{eff}}(\mathbf{r})\right] \psi_{\mathbf{k}, i}(\mathbf{r})+\hbar \int d^{3} \mathbf{r}^{\prime} \widetilde{\Sigma}\left(\mathbf{r}, \mathbf{r}^{\prime} ; E_{\mathbf{k}, i}^{\mathrm{qp}}\right) \psi_{\mathbf{k}, i}\left(\mathbf{r}^{\prime}\right)} \\
& \quad=E_{\mathbf{k}, i}^{\mathrm{qp}} \psi_{\mathbf{k}, i}(\mathbf{r}) .
\end{aligned}
$$

Here $\widetilde{\Sigma}$ is the electron self-energy corrected by the correlation effects already included in the effective potential of the $\mathrm{KS}$ equations via $v_{\mathrm{xc}}(\mathbf{r})$. Since the $e$-ph interaction is treated separately, $\widetilde{\Sigma}$ only contains contributions due to the $e$-e interaction. The state-of-the-art approximation for the selfenergy for weakly correlated electron systems of real materials is the $G W$ approximation, ${ }^{33,45}$ in which the self-energy is obtained from the product of the single-electron Green's function corresponding to the $\mathrm{KS}$ equations (5) and the screened potential $W$,

$$
\widetilde{\Sigma}\left(\mathbf{r}, \mathbf{r}^{\prime} ; t\right)=i G_{\mathrm{LDA}}\left(\mathbf{r}, \mathbf{r}^{\prime} ; t\right) W\left(\mathbf{r}, \mathbf{r}^{\prime} ; t^{+}\right)-v_{\mathrm{xc}}(\mathbf{r}) \delta\left(\mathbf{r}-\mathbf{r}^{\prime}\right) .
$$

$W$ is the pure Coulomb potential $v$ screened by the dielectric function $\epsilon$, in symbolic notation $W=v / \epsilon$. Once the Dyson equation is solved, the $e$-e contribution to the lifetime $\tau$, or the linewidth $\Gamma$, respectively, can be obtained-as in the phonon case-from the imaginary part of the electron selfenergy evaluated at the real part of the quasiparticle energy $E_{\mathbf{q}, i}$

$$
\hbar / \tau_{e-e}\left(E_{\mathbf{k}, i}^{\mathrm{qp}}\right)=\Gamma_{e-e}\left(E_{\mathbf{k}, i}^{\mathrm{qp}}\right)=-2 \hbar \operatorname{Im} \widetilde{\Sigma}_{i, i}\left(\mathbf{k}, E_{\mathbf{k}, i}^{\mathrm{qp}}\right) .
$$

Since in the Green's function formalism the adding or removing of an electron are equivalent procedures, the calculation of the lifetime of excited electrons or holes bears no difference.

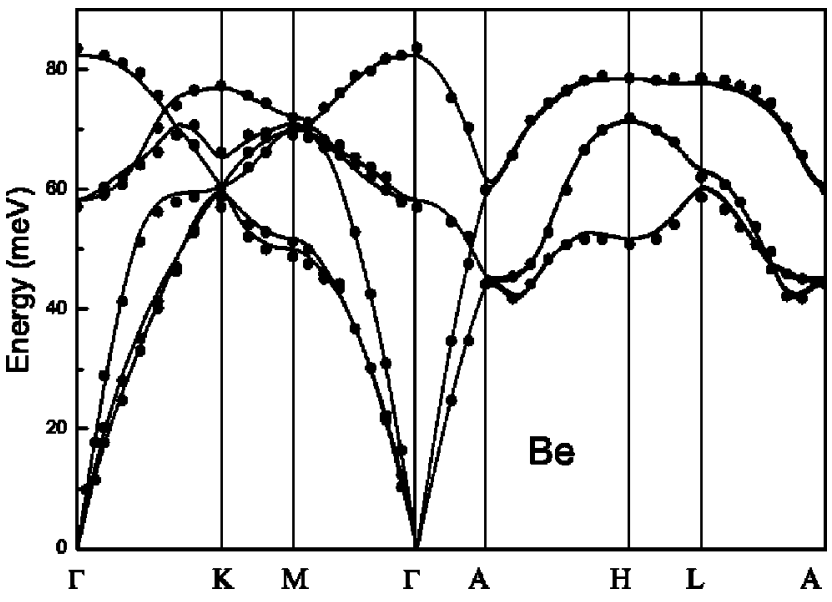

FIG. 2. Calculated phonon dispersion curves along the highsymmetry directions for hcp Be. The filled circles indicate the experimentally measured frequencies (Ref. 48).

All the numbers quoted in this article for the linewidth due to electron-electron interaction were obtained from a full solution of Eqs. (5)-(8). For comparison we also calculate the $\Gamma_{e-e}$ values in some symmetry points of the BZ at the LDA eigenvalues ("energy-shell" approximation).

\section{RESULTS AND DISCUSSION}

\section{A. Electron-phonon interaction}

In the linear response calculation of the phonon modes we used 120 special $\mathbf{k}$ points in the irreducible wedge of the Brillouin zone (IBZ) and the first-order Hermite-Gaussian smearing technique ${ }^{46}$ with a smearing width of $0.02 \mathrm{Ry}$, leading to energy bands as shown in Fig. 1. The evaluated phonon spectrum along the symmetry directions of BZ is shown in Fig. 2. Our results are similar to those obtained in the earlier DFPT calculation for bulk Be (Ref. 47) and are in good agreement with the experimental neutron diffraction data. ${ }^{48}$ To obtain the spectral functions needed for the calculation of the imaginary part of the self-energy the summation over the phonon wave vectors $\mathbf{q}$ and phonon branches $\nu$ was performed by using the linear tetrahedron method ${ }^{49}$ with more than 6000 tetrahedra in the IBZ because of rather significant variation of the electron-phonon matrix elements throughout the BZ. The Dirac delta functions in Eqs. (2) and (4) containing the electron band energies have been approximated by first-order Hermite-Gaussian functions with a smearing width in the range of 0.01-0.03 Ry.

In Fig. 3 we show Eliashberg spectral function $\alpha^{2} F(w)$ averaged over the Fermi surface, which measures the contribution of phonons with energy $w$ to scattering processes at the Fermi surface. To calculate the double Fermi surface sums in Eq. (4) properly we used a dense mesh of up to $30000 \mathbf{k}$ points in the IBZ because of a very small and complicated Fermi surface in Be. Since the set of wave vectors sampled in the IBZ is very large the results are almost insensitive to the value of the smearing width. There are no experimental data for $\alpha^{2} F(w)$ in Be. This function is determined to a large extent by the phonon density of states $F(w)$ 


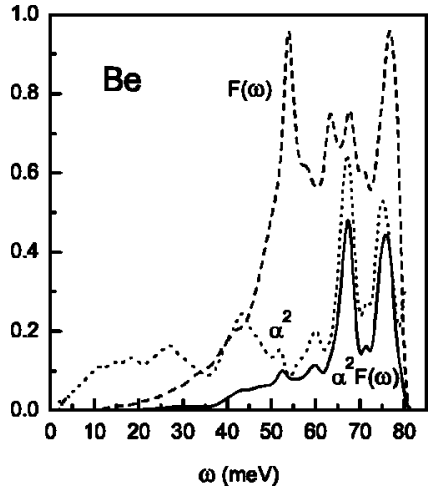

FIG. 3. Phonon density of states $F(w)$ (dashed line) and electron-phonon spectral function $\alpha^{2} F(w)$ averaged over the Fermi surface (solid line) for Be. The coupling function $\alpha^{2}$ [defined as the ratio $\left.\alpha^{2} F(w) / F(w)\right]$ is shown by dotted line.

(also shown in Fig. 3). Nevertheless, in the case of Be the two curves are rather different in shape, especially in the lower frequency region. The Eliashberg spectral function is dominated by two large peaks at high frequencies around $w=67.4 \mathrm{meV}$ and $w=76 \mathrm{meV}$. These peaks are mainly determined by the scattering of the two lower and the upper optical phonons, correspondingly, from the $K$ point region. The lower-frequency peak in the density of states $F(w)$ is completely suppressed by the electron-phonon matrix elements. The corresponding electron-phonon mass enhancement parameter $\left(\lambda\right.$ at $\left.E_{F}\right)$ is found to be small, $\lambda=0.21$. This confirms that for Be the electron-phonon coupling is rather weak as it was pointed out in the earlier reported theoretical estimates of $\lambda$ based on semiempirical relations to the critical temperature and pseudopotential calculations $\lambda$ $=0.24 \pm 0.05 .^{1,30-32}$

Let us consider now how $\mathbf{k}$-selected electron states couple to phonons and how this coupling varies with $\mathbf{k}$. In Fig. 4 we show the Eliashberg spectral functions $\alpha^{2} F_{\mathbf{k}, i}(w)$ for some high symmetry points in the IBZ [Eq. (2)]. The results are shown for $\Gamma_{3}^{+}$(solid line), $\Gamma_{4}^{-}$(dashed line) electron states [Fig. 4(a)], and for the second and the third electron states at $H$ [Fig. 4(c)] and $K$ [Fig. 4(d)]. At the Fermi level [Fig. 4(b)] the electron-phonon spectral function is given for $\Sigma_{3}$ (solid line) and $\Sigma_{1}$ (dashed line) energy bands in the $\Gamma M$ direction (see Fig. 1). As follows from the figure the $\lambda_{\mathbf{k}, i}$ and $\alpha^{2} F_{\mathbf{k}, i}(w)$ for $\Gamma_{4}^{-}$and the corresponding energy band $\Sigma_{1}$ at the Fermi level do not differ substantially while for $\Gamma_{3}^{+}$and the corresponding band $\Sigma_{3}$ at $E_{F}$ these quantities increase notably on moving from $\Gamma$ to the Fermi level. For these electron states the strong $e$-ph coupling is observed at high phonon energies $(60-80 \mathrm{meV})$. In the case of the $\Gamma_{3}^{+}$and $\Gamma_{4}^{-}$electron states the main contribution comes from optical phonons near the $L$ and $H$ symmetry points as well as from the $\Gamma K$ direction. For the states at the Fermi level only optical modes in the $\Gamma K$ direction and the two upper optical phonons along $\Gamma M$ contribute to the scattering processes. However, the electronphonon coupling in these states is rather small compared to electron states at other symmetry points. At $H$ and $K$ there is an enhanced spectral weight at lower frequencies around $\omega=55 \mathrm{meV}$, which is completely suppressed for the electron
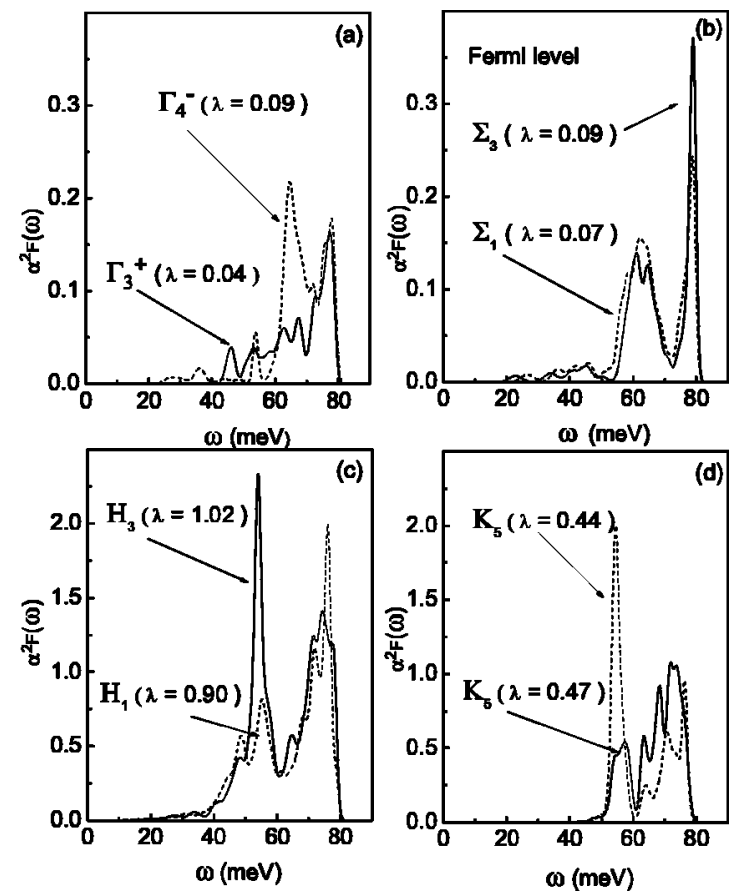

FIG. 4. The electron-phonon spectral function $\alpha^{2} F_{\mathbf{k}, i}(w)$ is shown for some electron states at the $\Gamma$ (a), $H$ (c), $K$ (d) symmetry points and at the Fermi level (b) in the $\Gamma M$ direction.

states in the $\Gamma M$ direction. This peak is mainly determined by the scattering of the two lower optical phonons near the $\Gamma$ point and the two upper optical modes at $A$. For the unoccupied electron state at $H$ the only contribution comes from the lower optical mode near the $L$ point. The high-energy peaks are determined by interactions with optical vibrations mainly from the region of the $H$ point. Since $\lambda_{\mathbf{k}, i}$ is defined as the inverse moment of $\alpha^{2} F_{\mathbf{k}, i}(w)$ the differences in the Eliashberg spectral function lead to considerable differences in $\lambda_{\mathbf{k}, i}$ at these points. While at the $\Gamma$ point $\lambda$ is very small for both states considered $\left(\lambda_{\Gamma_{3}^{+}}=0.04, \lambda_{\Gamma_{4}^{-}}=0.09\right)$ and only increases slightly for the first electron state on moving to the Fermi level $\left(\lambda_{\Sigma_{3}}=0.09\right)$ the electron-phonon coupling at the $H$ and $K$ symmetry points enhances significantly with $\lambda_{H_{3}}=1.02$, $\lambda_{H_{1}}=0.9$, and $\lambda_{K_{5}}^{1}=0.47, \lambda_{K_{5}}^{2}=0.44$, respectively. Thus the value of $\lambda$ depends strongly on the position of the wave vector.

Using the calculated spectral functions $\alpha^{2} F_{\mathbf{k}, i}(w)$ we evaluated the electron-phonon contribution $\Gamma_{e \text {-ph }}$ to the total linewidth of electrons (holes) as a function of energy. The top panel of Fig. 5 gives the energy dependence of the lifetime broadening calculated at $T=0$ for $\Sigma_{3}$ and $\Sigma_{1}$ energy bands in the $\Gamma M$ direction. The middle panel of the figure shows the variation of the electron-phonon coupling parameter $\lambda$ for the same energies measured from the Fermi level. As follows from the figure, $\Gamma_{e \text {-ph }}$ varies fast around $E_{F}$ for electron energies smaller than the maximum phonon frequency and on moving from the $\Gamma$ point to the Fermi level (negative electron energies for the $\Sigma_{3}$ band and positive energies for the $\Sigma_{1}$ band). In the latter case $\Gamma_{e \text {-ph }}$ first increases by a factor of 3 for both energy bands (a), (b) and then it achieves a minimum in the center of the $\Gamma M$ direction. Near 

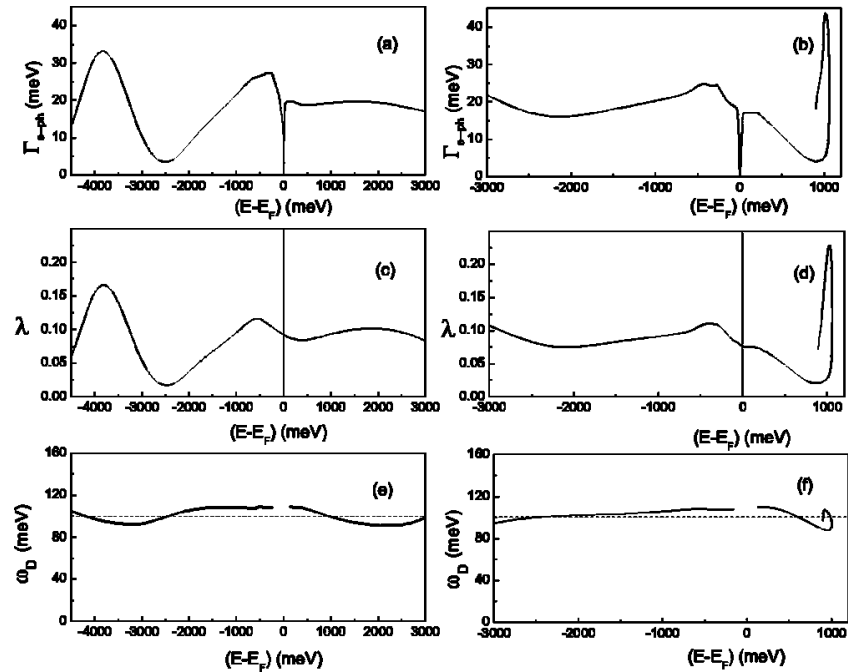

FIG. 5. The $T=0$ phonon induced lifetime broadening $\Gamma_{e-p h}$ as a function of the energy for $\Sigma_{3}$ (a) and $\Sigma_{1}$ (b) energy bands in the $\Gamma M$ direction and the energy dependence of the electron-phonon coupling parameter $\lambda(\mathrm{c}),(\mathrm{d})$ as well as of the characteristic Debye frequency $\omega_{D}$ (e), (f) calculated from equation $\Gamma_{e-\mathrm{ph}}=2 \pi \lambda \hbar \omega_{D} / 3$ for these states.

the Fermi level, at the maximum phonon energy, new maxima of the $\Gamma_{e \text {-ph }}$ appear. However, when the energy increases from zero to that at the $M$ symmetry point the different behavior of the phonon-induced lifetime broadening is observed. As the energy exceeds the maximum phonon energy the $\Gamma_{e \text {-ph }}$ becomes almost saturated. The electronphonon enhancement parameter $\lambda_{\mathbf{k}, i}$ that reflects the variation of $\Gamma_{e-p h}$ does not change notably in the latter case and the values of $\lambda_{\mathbf{k}, i}$ at the $M$ symmetry point for these states $\left(\lambda_{M_{2}^{-}}=0.12\right.$ and $\left.\lambda_{M_{4}^{-}}=0.09\right)$ are almost equal to those at $E_{F}$. For the $\Sigma_{1}$ energy band above the Fermi level the data also show an additional $\mathbf{k}$ dependence of the electron-phonon coupling parameter because we have the different values of $\lambda_{\mathbf{k}, i}$ on both sides of the energy maximum seen in Fig. 1. These results confirm that both the $\Gamma_{e \text {-ph }}$ and the $\lambda_{\mathbf{k}, i}$, depend indeed strongly on the $\mathbf{k}$ position and on the energy of a state.

Studying electron-phonon interaction in thin films of $\mathrm{Ag}$ on $\mathrm{Fe}(100)$ Paggel et al. $^{20}$ found that for quantum well states in $\mathrm{Ag} \lambda$ varies from 0.015 to 0.52 . They attributed this difference to the symmetry of electron states. The lower value was obtained for $d$-quantum well states and the largest one for $s p$ states. In the case of Be all the states considered are of the $s p$ symmetry, nevertheless, even the bigger spread of the $\lambda$ values is obtained for this material.

From the calculated $\Gamma_{e \text {-ph }}$ and $\lambda$ as functions of energy the characteristic Debye frequency $\omega_{D}$ is evaluated by using the relation $\Gamma_{e-\mathrm{ph}}=2 \pi \lambda \hbar \omega_{D} / 3$ derived within the Debye model at $T=0 \mathrm{~K}$ for electron (hole) energies $\left|\epsilon-E_{F}\right|>\hbar \omega_{D}{ }^{1,2,50}$ The energy dependence of $\omega_{D}$ is shown in the bottom panel of Fig. 5. We do not show $\omega_{D}$ for $\left|\epsilon-E_{F}\right|<\hbar \omega_{D}$ since for these energies $\Gamma_{e \text {-ph }}$ shows nonlinear dependence on energy. ${ }^{1}$ As one can see for both energy bands considered the Debye frequency depends very slightly on energy. Even for the $\Sigma_{1}$ band at about $1000 \mathrm{meV}$ above $E_{F}$ where the band is very

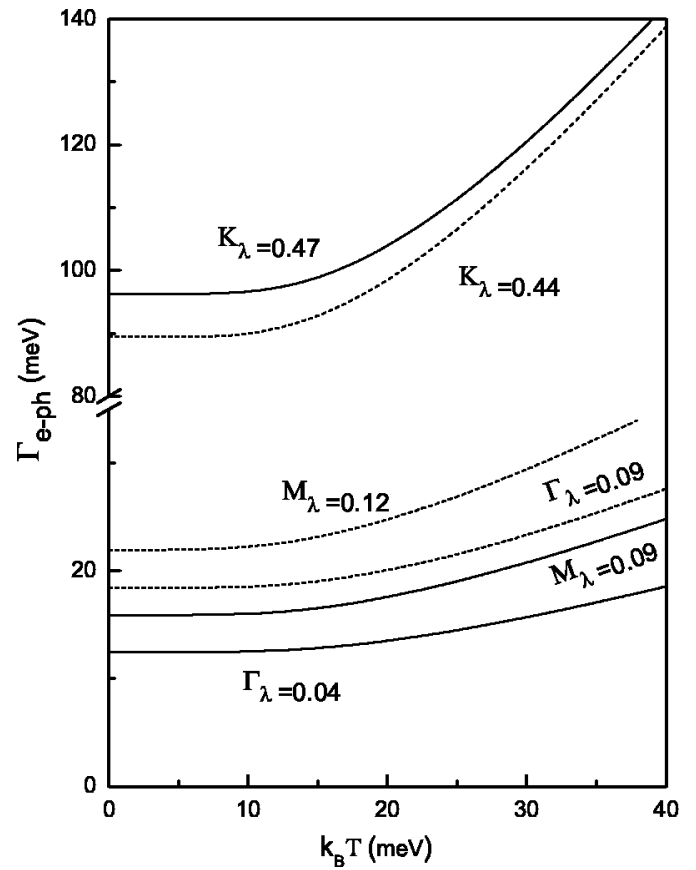

FIG. 6. The temperature dependence of the phonon induced lifetime broadening for the $\Gamma_{3}^{+}, K_{5}^{1}, M_{4}^{-}$(solid lines) and the $\Gamma_{4}^{-}, K_{5}^{2}, M_{2}^{-}$ (dashed lines) electron states.

flat and demonstrates the extraordinary dependence of $\Gamma_{e \text {-ph }}$ and $\lambda$ on energy the variation of $\omega_{D}$ does not exceed $10 \%$ of the averaged value. The averaged $\omega_{D}$ (dashed lines) for both the $\Sigma_{3}$ and $\Sigma_{1}$ bands is equal to $100 \pm 10 \mathrm{meV}$. This value agrees fairly well with the experimental $\omega_{D}$ values of $86 \mathrm{meV}$ (Ref. 51) and $124 \mathrm{meV} .{ }^{52}$ The obtained result demonstrates that despite very serious approximations used, the Debye model can give a reasonable description of $\Gamma_{e \text {-ph }}$ for real bulk metals provided that $\lambda_{\mathbf{k}, i}$ and $\omega_{D}$ are known from experiments or from first-principle calculations. This conclusion is also confirmed by numerous measurements of $\lambda$ and $\Gamma_{e \text {-ph }}$ for surface states on metals ${ }^{4-14}$ and for quantum well states in thin films. ${ }^{15-20}$

We also studied a temperature dependence of the phonon induced lifetime broadening for the second and the third electron states at the $\Gamma, K$ and $M$ symmetry points as shown in Fig. 6. At $T=0$ we only have phonon emission scattering processes. For temperatures $k_{B} T$ higher than the maximum phonon frequency the $T$ dependence of $\Gamma_{e \text {-ph }}$ becomes linear with a slope, which is determined by the electron-phonon coupling parameter $\lambda$. For electron states at the $\Gamma$ and $M$ points the values of $\lambda$ do not differ notably and the slopes of the corresponding curves are almost the same while for states at the $K$ point the slope is steepper reflecting the larger values of $\lambda_{\mathbf{k}, i}$.

\section{B. Electron-electron interaction}

The results presented here for the electron-electron contribution to the total linewidth are based on calculations performed along the lines of Sec. II B. The Brillouin zone was sampled with a $16 \times 16 \times 12$ mesh and the plane-wave cutoff used was 20 Ry. The internal summations necessary for cal- 

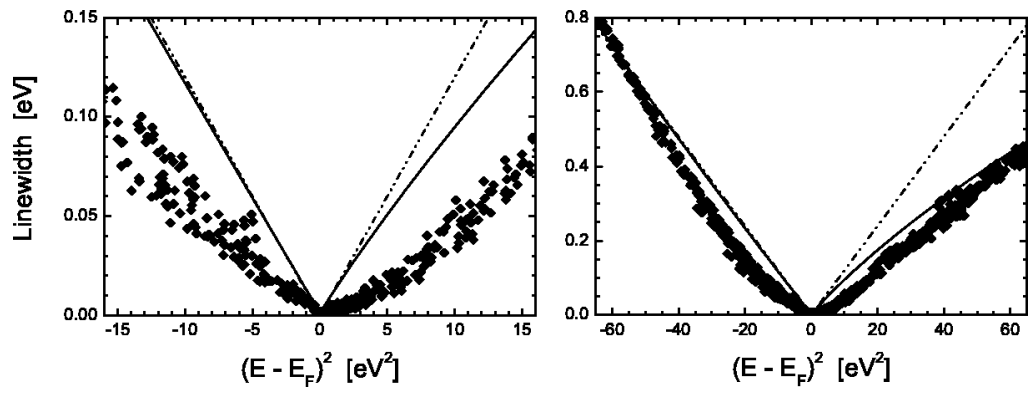

FIG. 7. The linewidth $\Gamma_{e-e}$ of holes and excited electrons for $\mathrm{Be}$ is given versus the square of the energy. In the left panel $\Gamma_{e-e}$ is shown (diamonds) for states located within $4 \mathrm{eV}$ of $E_{F}$ and in the right panel $\Gamma_{e-e}$ is given for states within $8 \mathrm{eV}$ of $E_{F}$. The double-dotted-dashed curve is the result of Eq. (9) for a Wigner-Seitz radius of $r_{s}=1.87$. The solid line is the result of a full jellium $G W$ calculation for the same value of $r_{s}$. culating the electron self-energy included 20 bands, meaning that an energy range of up to $50 \mathrm{eV}$ above the Fermi energy was covered. Using these parameters the real part of the quasiparticle energy is converged to within $50 \mathrm{meV}$.

In the previous paragraph the discussion focused on relatively small excitation energies, where the $e$-ph contribution to the lifetime is significant compared to the $e$ - $e$ one. Since for larger excitation energies the contributions to the linewidth due to electron-electron scattering dominate, both energy ranges are covered in the following discussion. Figure 7 shows the calculated linewidth as diamonds for all electron states in the energy range of $4 \mathrm{eV}$ around the Fermi energy (left panel) and up to $8 \mathrm{eV}$ in the plot on the right-hand side. In this figure the data for all electron states which were used in the calculation of the self-energy are collected. The figure clearly shows that the linewidth for small excitation energies cannot be described by a simple law or model as in the case of the homogeneous electron gas (HEG, jellium model). The linewidth does increase with increasing distance from the Fermi energy but the data are not on a straight line but are scattered over a significant range. The scattering decreases for energies of more than $6 \mathrm{eV}$ away from the Fermi energy.

The wide range of linewidths for different states of the same energy is a result of the band structure of Be as shown in Fig. 1. The band structure reveals bands, which resemble quite well the parabola of the free electron gas. But Be also has flat bands, which look similar to the $d$ bands of transition metals. In addition Be has energy gaps in many directions of the band structure. This results in a free-electron-like density of states for the lowest occupied states, but a dip across the Fermi energy. It is obvious that the linewidth of Be cannot be described by a HEG model for states in the energy region of approximately -4 to $4 \mathrm{eV}$.

It is, however, interesting to explore to which extent the jellium model gives reliable results for states above and below the just mentioned energy range. The direct way is the evaluation of Eqs. (6)-(8) within the jellium model as first done by Hedin. ${ }^{33}$ The result of such a calculation for a Wigner-Seitz radius of $r_{s}=1.87$ is given by the solid lines in Fig. 7. This value corresponds to the electron density $n$ $=3 /\left(4 \pi r_{s}^{3}\right)$ obtained from the lattice constants of real Be. For higher electron energies this conclusion was confirmed in Ref. 53.

Despite the fact that the isotropic jellium model is fairly simple such computations are complex. Therefore very often an approximation is utilized, in which a low-frequency expansion of the dielectric function $\epsilon$ is made. This leads to a closed expression for the linewidth of holes and excited electrons within the $\mathrm{HEG},{ }^{54,55}$

$$
\Gamma=\Gamma_{0}\left(E-E_{F}\right)^{2} \quad \text { with } \quad \Gamma_{0}=\frac{\pi^{2}}{64 E_{F}} \sqrt{\frac{3 \pi n e^{2}}{m}}
$$

In the literature Eq. (9) is often referred to as Fermi liquid theory (FLT). The double-dotted-dashed lines in Fig. 7 denote the result of Eq. (9) for $r_{s}=1.87$.

The comparison of the two jellium results with the $a b$ initio data in Fig. 7 confirms that the HEG cannot be used in order to describe Be within say $4 \mathrm{eV}$ of the Fermi energy. In this energy range calculations have to be performed, which take the true band structure of Be into account. On the other hand the dot-dot-dashed and solid curves agree quite well with the $a b$ initio data for larger energies. The two jellium results also illustrate nicely that the so-called FLT can only be applied to states close to the Fermi energy. For higher excited electrons the results of Eq. (9) differ quite substantially from those of the full jellium calculation, which is due to the asymmetry of the imaginary part of the self-energy with respect to energy. 56

The results of the above discussion are bolstered by a wave-vector resolved view on the data. Figure 8 shows the wave-vector resolved linewidth plotted versus the square of the energy measured from the Fermi energy for four different directions $\Gamma-M, M-L, L-A$, and $A-\Gamma$ for an energy range covering the occupied bands (holes) and the first two conduction
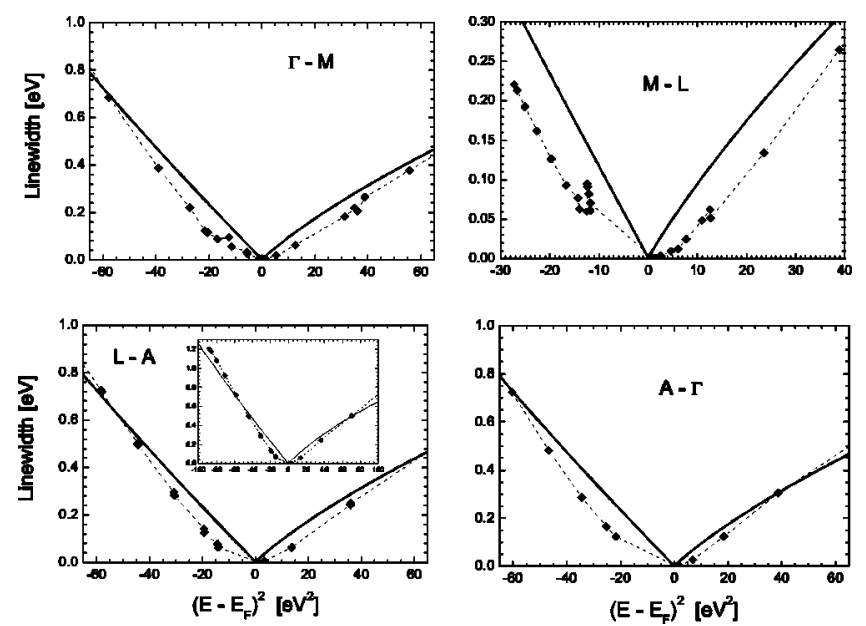

FIG. 8. Linewidth of holes and excited electrons due to electronelectron scattering in Be plotted versus the energy measured from the Fermi energy for wave vectors from the different directions in the BZ. Each plot contains the data (filled diamonds) for the first four bands. The dashed lines are a guide to the eye. The solid lines denote the result of a full jellium $G W$ calculation for $r_{s}=1.87$. 

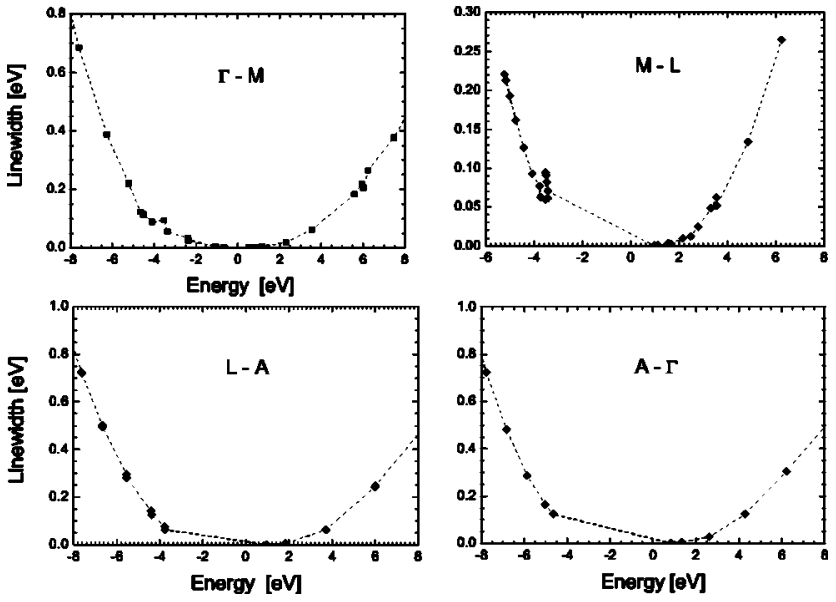

FIG. 9. As Fig. 8 but here the linewidth is plotted linearly versus the energy.

bands (excited electrons). In the sequence given above the chosen directions describe a rectangle in reciprocal space. For each direction the obtained linewidths are marked by filled diamonds. The dashed curves serve as a guide to the eye. As a comparison the result of the full jellium $G W$ calculation are given as solid lines.

Figure 9 shows the same data but now plotted linearly in energy. The two figures show that although the impact of the four different bands entering each plot is quite different for the four directions it is in all directions clearly noticable. In Fig. 10 the band dependence is disentangled and magnified at the example of the $\Sigma_{1}$ and $\Sigma_{3}$ bands in the $M-\Gamma$ direction (see Fig. 1). Below the Fermi energy both bands resemble to a certain extent the parabola of a HEG model and both bands cross the Fermi energy. In Fig. 10 this is reflected by very similar data for the lifetimes of holes in these bands. In the range of excited electrons, however, $\Sigma_{3}$ keeps its character, while $\Sigma_{1}$ becomes almost flat. Consequently the lifetimes for excited electrons in these two bands are very different.

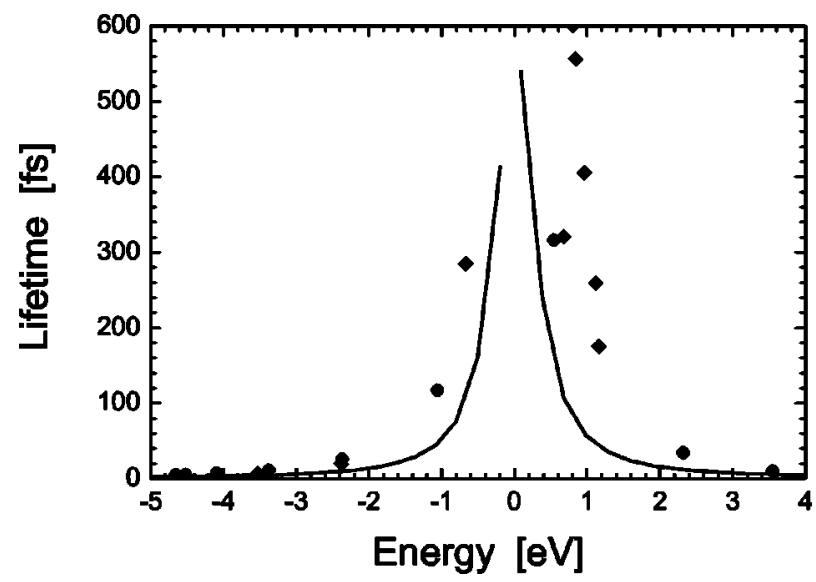

FIG. 10. The lifetime of holes and excited electrons for the bands $\Sigma_{1}$ (diamonds) and $\Sigma_{3}$ (circles) in fs plotted versus the energy measured from the Fermi energy. The solid line denotes the result for a full jellium $G W$ calculation for $r_{s}=1.87$.
TABLE I. The total linewidth $\Gamma_{\text {tot }}$, electron-phonon $\Gamma_{e-\mathrm{ph}}$, and electron-electron $\Gamma_{e-e}$ contributions for certain states at the $\Gamma, M$, and $L$ points. $\Gamma_{e-e}$ is given both at the quasiparticle and at the LDA (in parentheses) energies. The second column denotes the LDA eigenvalue.

\begin{tabular}{ccccc}
\hline \hline State & $\begin{array}{c}\epsilon^{\mathrm{LDA}} \\
{[\mathrm{eV}]}\end{array}$ & $\begin{array}{c}\Gamma_{e-\mathrm{ph}} \\
{[\mathrm{meV}]}\end{array}$ & $\begin{array}{c}\Gamma_{e-e} \\
{[\mathrm{meV}]}\end{array}$ & $\begin{array}{c}\Gamma_{\text {tot }} \\
{[\mathrm{meV}]}\end{array}$ \\
\hline$\Gamma_{3}^{+}$ & -4.28 & 13 & $124(124)$ & 137 \\
$\Gamma_{4}^{-}$ & 1.19 & 18 & $1(1)$ & 19 \\
$M_{1}^{+}$ & -4.87 & 40 & $221(189)$ & 261 \\
$M_{2}^{-}$ & -3.07 & 22 & $95(84)$ & 117 \\
$M_{4}^{-}$ & 3.54 & 16 & $62(80)$ & 78 \\
$L_{1}$ & -3.54 & 38 & $77(78)$ & 115 \\
$L_{1}$ & 1.55 & 76 & $1(3)$ & 77 \\
\hline \hline
\end{tabular}

\section{General discussion}

Having discussed the linewidth of holes and excited electrons separately for $e$-ph and $e-e$ scattering we can now compare the two sets of numbers. In Table I the values of the phonon induced lifetime broadening at $T=0$ are compared with the $e-e$ contributions for some selected electron states at the $\Gamma, M$, and $L$ symmetry points. In general, the linewidth due to the $e$-ph scattering is smaller than that induced by the $e$-e interactions. For the electron states $\Gamma_{3}^{+}$and $M_{1}^{+}$the $e$-e contribution is nearly one order of magnitude larger. However, for some electron states, such as $\Gamma_{4}^{-}$and $L_{1}$ (above the Fermi level), $\Gamma_{e-\mathrm{ph}}$ is much bigger than $\Gamma_{e-e}$ since for these states the $e$-e contribution is very small. This is related to the absence of final electron states with relatively small momenta for the electron (hole) decay while for big momenta the decay probability is small. As for $e$-ph interaction, the big momenta scattering favors the large contribution to the $e$ ph coupling. This results in a small lifetime of these states in spite of the negligible values of the $e$ - $e$ scattering. For higher temperatures the changes in the $e$-ph contribution depend on the value of the $e$-ph mass enhancement parameter $\lambda_{\mathbf{k}, i}$. As a rule they are not very large, which is consistent with the rather weak $e$-ph coupling that is observed in hcp Be. Thus, at room temperature the $e$-ph contributions to the linewidth for $M_{1}^{+}, M_{2}^{-}, M_{4}^{-}$electron states are found to be 48,28 , and $20 \mathrm{meV}$, respectively. As one can see they remain smaller than the linewidths due to the $e-e$ scattering for the same states, which do not depend on temperature. In Table I we also compare $\Gamma_{e-e}$ evaluated at the quasiparticle energies with $\Gamma_{e-e}$ computed at the LDA eigenvalues (an "energy-shell" approximation). The latter are shown in parentheses. One can see that the "energy-shell" $\Gamma_{e-e}$ values are in a fairly good agreement with those obtained at the quasiparicle energies.

\section{SUMMARY AND CONCLUSIONS}

A first-principles study of the electron-phonon and electron-electron contributions to the lifetime broadening for electron states in bulk Be has been presented. The obtained results for the phonon-induced linewidth $\Gamma_{e-\mathrm{ph}}$ and for the electron-phonon coupling parameter $\lambda$ show a strong depen- 
dence on the $\mathbf{k}$ position and on the energy of a state. In particular, $\lambda$ varies from 0.01 to 1.02 as a function of electron energy and momentum, while $\lambda=0.21$ as averaged over momenta at the Fermi surface has been obtained. The latter value is consistent with the earlier reported theoretical estimates of $\lambda$ based on semiempirical relations to the critical temperature $T_{c}$ and pseudopotential calculations. It has been shown that the $e$-ph coupling matrix elements strongly affect the Eliashberg function especially for low phonon frequencies. The utility of the Debye model for evaluations of the $\Gamma_{e \text {-ph }}$ for a real material was demonstrated by using the Debye model relation between the calculated $\Gamma_{e \text {-ph }}$ and $\lambda$, and the characteristic Debye frequency $\omega_{D}$ has been obtained in good agreement with the experimental $\omega_{D}$. For band energies
$\left|\epsilon-E_{F}\right|>\hbar \omega_{D}$ the electron induced linewidth $\Gamma_{e-e}$ as a rule is significantly larger than the $\Gamma_{e-\mathrm{ph}}$. However, for some states the $\Gamma_{e \text {-ph }}$ is one order of magnitude bigger than the $\Gamma_{e-e}$.

\section{ACKNOWLEDGMENTS}

W.-D.S. gratefully acknowledges G. Ertl for his interest and generous support. This work was partially supported by MCyT (Grant No. MAT 2001-0946) and the European Community 6th Network of Excellence NANOQUANTA (Grant No. NMP4-CT-2004-500198). W.-D.S. was supported by the Deutsche Forschungsgemeinschaft through Grant No. SFB 450 .
*Electronic address: waptctce@sq.ehu.es

${ }^{1}$ G. Grimvall, The Electron-Phonon Interaction in Metals (NorthHolland, Amsterdam, 1981).

${ }^{2}$ P. M. Echenique, R. Berndt, E. V. Chulkov, Th. Fauster, A. Goldmann, and U. Höfer, Surf. Sci. Rep. 52, 219 (2004).

${ }^{3}$ B. Hellsing, A. Eiguren, and E. V. Chulkov, J. Phys.: Condens. Matter 14, 5959 (2002).

${ }^{4}$ B. A. McDougall, T. Balasubramanian, and E. Jensen, Phys. Rev. B 51, R13 891 (1995).

${ }^{5}$ R. Matzdorf, G. Meister, and A. Goldmann, Phys. Rev. B 54, 14807 (1996).

${ }^{6}$ R. Matzdorf, Surf. Sci. Rep. 30, 153 (1998).

${ }^{7}$ T. Balasubramanian, E. Jensen, X. L. Wu, and S. L. Hulbert, Phys. Rev. B 57, R6866 (1998).

${ }^{8}$ T. Valla, A. V. Fedorov, P. D. Johnson, and S. L. Hulbert, Phys. Rev. Lett. 83, 2085 (1999).

${ }^{9}$ T. Balasubramanian, P.-A. Glans, and L. I. Johansson, Phys. Rev. B 61, 12709 (2000).

${ }^{10}$ A. Eiguren, B. Hellsing, F. Reinert, G. Nicolay, E. V. Chulkov, V. M. Silkin, S. Hüfner, and P. M. Echenique, Phys. Rev. Lett. 88, 066805 (2002).

${ }^{11}$ S.-J. Tang, Ismail, P. T. Sprunger, and E. W. Plummer, Phys. Rev. B 65, 235428 (2002).

${ }^{12}$ J. E. Gayone, S. V. Hoffmann, Z. Li, and Ph. Hofmann, Phys. Rev. Lett. 91, 127601 (2003).

${ }^{13}$ M. Hengsberger, D. Purdie, P. Segovia, M. Garnier, and Y. Baer, Phys. Rev. Lett. 83, 592 (1999).

${ }^{14}$ S. LaShell, E. Jensen, and T. Balasubramanian, Phys. Rev. B 61, 2371 (2000).

${ }^{15}$ A. Carlsson, B. Hellsing, S.-Å. Lindgren, and L. Walldén, Phys. Rev. B 56, 1593 (1997).

${ }^{16}$ T.-C. Chiang, Surf. Sci. Rep. 39, 181 (2000).

${ }^{17}$ T. Valla, M. Kralj, A. Šiber, M. Milun, P. Pervan, P. D. Johnson, and D. P. Woodruff, J. Phys.: Condens. Matter 12, L477 (2000).

${ }^{18}$ M. Kralj, A. Šiber, P. Pervan, M. Milun, T. Valla, P. D. Johnson, and D. P. Woodruff, Phys. Rev. B 64, 085411 (2001).

${ }^{19}$ D.-A. Luh, T. Miller, J. J. Paggel, and T.-C. Chiang, Phys. Rev. Lett. 88, 256802 (2002).

${ }^{20}$ J. J. Paggel, D.-A. Luh, T. Miller, and T.-C. Chiang, Phys. Rev. Lett. 92, 186803 (2004).

${ }^{21}$ N. V. Smith, P. Thiry, and Y. Petroff, Phys. Rev. B 47, 15476
(1993).

${ }^{22}$ G. M. Eliashberg, Zh. Eksp. Teor. Fiz. 38, 966 (1960) [Sov. Phys. JETP 11, 696 (1960)].

${ }^{23}$ G. M. Eliashberg, Zh. Eksp. Teor. Fiz. 43, 1005 (1962) [Sov. Phys. JETP 16, 780 (1962)].

${ }^{24}$ I. Campillo, V. M. Silkin, J. M. Pitarke, E. V. Chulkov, A. Rubio, and P. M. Echenique, Phys. Rev. B 61, 13484 (2000).

${ }^{25}$ D. A. Papaconstantopoulos, Handbook of Band Structure of Elemental Solids (Plenum, New York, 1986).

${ }^{26}$ E. V. Chulkov, V. M. Silkin, and E. N. Shirykalov, Surf. Sci. 188, 287 (1987).

${ }^{27}$ V. M. Silkin and E. V. Chulkov, Phys. Solid State 37, 1540 (1995) [Fiz. Tverd. Tela (S.-Petersburg) 37, 2795 (1995)].

${ }^{28} \mathrm{Ph}$. Hofmann, R. Stumpf, V. M. Silkin, E. V. Chulkov, and E. W. Plummer, Surf. Sci. 355, L278 (1996).

${ }^{29}$ A. Eiguren, S. de Gironcoli, E. V. Chulkov, P. M. Echenique, and E. Tosatti, Phys. Rev. Lett. 91, 166803 (2003).

${ }^{30}$ W. L. McMillan, Phys. Rev. 167, 331 (1968).

${ }^{31}$ J. W. Garland and P. B. Allen, Physica (Amsterdam) 55, 669 (1971).

${ }^{32}$ P. B. Allen and M. L. Cohen, Phys. Rev. 187, 525 (1969).

${ }^{33}$ L. Hedin, Phys. Rev. 139, A796 (1965).

${ }^{34}$ S. Baroni, S. de Gironcoli, A. D. Corso, and P. Giannozzi, Rev. Mod. Phys. 73, 515 (2001).

${ }^{35}$ S. Baroni, S. de Gironcoli, A. D. Corso, and P. Giannozzi, URL http://www.pwscf.org

${ }^{36}$ N. Troullier and J. L. Martins, Phys. Rev. B 43, 1993 (1991).

${ }^{37}$ J. P. Perdew and A. Zunger, Phys. Rev. B 23, 5048 (1981).

${ }^{38}$ W.-D. Schöne, R. Keyling, M. Bandić, and W. Ekardt, Phys. Rev. B 60, 8616 (1999).

${ }^{39}$ I. Campillo, J. M. Pitarke, A. Rubio, E. Zarate, and P. M. Echenique, Phys. Rev. Lett. 83, 2230 (1999).

${ }^{40}$ P. M. Echenique, J. M. Pitarke, E. V. Chulkov, and A. Rubio, Chem. Phys. 251, 1 (2000).

${ }^{41}$ V. P. Zhukov, F. Aryasetiawan, E. V. Chulkov, I. G. de Gurtubay, and P. M. Echenique, Phys. Rev. B 64, 195122 (2001).

${ }^{42}$ A. Marini, R. Del Sole, A. Rubio, and G. Onida, Phys. Rev. B 66, 161104(R) (2002)

${ }^{43}$ W. Kohn and L. J. Sham, Phys. Rev. 140, A1133 (1965).

${ }^{44}$ A. L. Fetter and J. D. Walecka, Quantum Theory of ManyParticle Systems (McGraw-Hill, New York, 1971). 
${ }^{45}$ F. Aryasetiawan and O. Gunnarsson, Rep. Prog. Phys. 61, 237 (1998).

${ }^{46}$ M. Methfessel and A. T. Paxton, Phys. Rev. B 40, 3616 (1989).

${ }^{47}$ M. Lazzeri and S. de Gironcoli, Surf. Sci. 402-404, 715 (1998).

${ }^{48}$ R. Stedman, Z. Amilius, R. Pauli, and O. Sundin, J. Phys. F: Met. Phys. 6, 157 (1976).

${ }^{49}$ P. E. Blöchl, Phys. Rev. B 50, 17953 (1994).

${ }^{50}$ E. V. Chulkov, J. Kliewer, R. Berndt, V. M. Silkin, B. Hellsing, S. Crampin, and P. M. Echenique, Phys. Rev. B 68, 195422 (2003).

${ }^{51}$ N. W. Ashcroft and N. D. Mermin, Solid State Physics (Saunders
College Publishing, Fort Worth, TX, 1976).

${ }^{52}$ C. Kittel, Introduction to Solid State Physics (Wiley, New York, 1976).

${ }^{53}$ V. M. Silkin, E. V. Chulkov, and P. M. Echenique, Phys. Rev. B 68, 205106 (2003).

${ }^{54}$ J. J. Quinn and R. A. Ferrell, Phys. Rev. 112, 812 (1958).

${ }^{55} \mathrm{D}$. Pines and P. Nozières, The Theory of Quantum Liquids (Addison-Wesley, Reading, MA, 1988).

${ }^{56}$ K. W.-K. Shung, B. E. Sernelius, and G. D. Mahan, Phys. Rev. B 36, R4499 (1987). 